\title{
Dislocation-Based Modeling and Numerical Analysis of Kink Deformations on the Basis of Linear Elasticity
}

\author{
Shunsuke Kobayashi* and Ryuichi Tarumi \\ Graduate School of Engineering Science, Osaka University, Osaka 560-8531, Japan
}

This study conducts dislocation-based modeling and numerical analysis on the deformation fields of two-dimensional kink structures. Peierls-Nabarro model is used to express edge dislocations in the elastic medium which are implemented into the weak form stress equilibrium equation using the extended isogeometric analysis. Numerical analysis revealed that the macroscopic deformations of two types of ortho kinks and four types of ridge kinks, $C_{2}^{+}, C_{2}^{-}, D_{1}^{+}$and $D_{1}^{-}$, agree well with the experimental results. Although most of the stress components are localized near the dislocation cores, only the component $\sigma_{22}$ in $C_{2}^{ \pm}$type ridge kinks showed broad distribution within the kink boundaries. On the other hand, deformation fields in $D_{1}^{ \pm}$type ridge kinks showed complementary rotation around the tip of kink structure. Such novel deformations would contribute to kink strengthening mechanism. [doi:10.2320/matertrans.MT-MM2019006]

(Received September 6, 2019; Accepted December 17, 2019; Published January 31, 2020)

Keywords: kink deformation, long-period stacking ordered magnesium alloys, Peierls-Nabarro model, extended isogeometric analysis

\section{Introduction}

Long-period stacking ordered magnesium (LPSO-Mg) has a dual phase microstructure consisting of intermetallic LPSO layer embedded in $\alpha$-Mg matrix. ${ }^{1)}$ After a hot extrusion of ascast ingot, this material shows notable mechanical properties such as high yield strength and fair elongation.,3) These properties are considered to be related to kink deformation which is different from conventional plastic deformation modes. In fact, recent experimental study suggests that this material shows the kink strengthening effect in addition to the conventional strengthening such as grain boundary refinement. ${ }^{4)}$ Understanding of the mechanism is, however, still in open and further investigation is required.

The first study on the kink deformation in metallic materials was reported by Orowan in 1942.5) In this paper, it is demonstrated that $\mathrm{Cd}$ single crystal shows novel kink deformation under uniaxial compressive loading where the loading axis is parallel to the basal plane of hcp crystal. Following to the pioneering study, several researchers confirmed the formation of kink structure in $\mathrm{Zn}$ single crystal. ${ }^{6-8)}$ In this case, uniaxial compression is also subjected parallel to the basal plane. Under the loading condition, Schmid factor of the primary slip systems of hcp crystals, i.e., $\langle 11 \overline{2} 0\rangle$ directions on the basal plane (0001), are identically zero. Hence, the basal dislocations are inactive during the uniform compressive deformation. On the other hand, the secondary slip systems or twinning are unable to move because of the strong plastic anisotropy of the hep crystals. Consequently, the single crystal specimen experiences elastic buckling before the activation of any plastic deformation and which induces non-uniform rotation of local crystallographic orientation. Such a rotation increases Schmid factor of the primary slip system and eventually induces the onedimensional motion of basal dislocations in the localized region so as to form the kink structure. This is the dislocation-based kink formation mechanism proposed by

*Graduate Student, Osaka University. Corresponding author, E-mail: kobayashi@nlsm.me.es.osaka-u.ac.jp
Hess and Barrett. ${ }^{6}$ This model explains the morphology as well as the structure of kink deformation from a phenomenological viewpoint.

Several materials, including LPSO-Mg alloys, polymers, minerals, composite materials and bundled papers, ${ }^{9-16)}$ show kink deformation in similar loading condition. A common feature of the materials is the strong plastic anisotropy due to layered structures. That is, the strong resistance to interlayer deformation restricts the effective plastic deformation to inner-layer deformation mode. Such a directional plastic deformation is analogous to the one-dimensional motion of basal dislocations in hcp crystals. It is, therefore, reasonable to suppose that the kink formation as well as the resulting strengthening in LPSO-Mg alloys are related to the plastic anisotropy due to the layered structure. More precisely, onedimensional motion of edge dislocations along the compressive direction plays an important role to understand the kink formation and resulting strengthening mechanism.

It is well known that interaction force between dislocations through stress fields are explained by the Peach-Koehler formula. ${ }^{17)}$ The stress fields due to kink-forming dislocations are, therefore, a clue for the understanding of kink strengthening mechanism. Up to date, atomistic-scale simulations have been conducted to reveal the kink formation process. ${ }^{18,19)}$ However, the stress fields around the kink structure has not been clarified yet.

This study aims to investigate the macroscopic deformation and internal stress fields of kink structure under several configuration of dislocations. Construction of the paper is as follows. In the next section, we introduce a two-dimensional elastic medium and consider the mechanics within the framework of the theory of linear elasticity and the calculus of variations. Edge dislocations are expressed by PeierlsNabarro mode ${ }^{17,20)}$ and introduced into the medium using extended isogeometric analysis (XIGA). ${ }^{21-24)}$ Note that XIGA is a generalization of IGA which solve the stress equilibrium equation in a weak form using the non-uniform rational B-spline (NURBS) basis function. ${ }^{25,26)}$ Results of numerical analysis are presented in Sec. 3. After a brief explanation on the simulation conditions, we present the 
results of numerical analysis including macroscopic deformation, elastic spin and two-dimensional stress components for several configurations of dislocations. Some concluding remarks are given in Sec. 4.

\section{Mathematical Modeling of Dislocations}

\subsection{Linear elasticity of dislocations}

Let $\Omega$ be a simply connected open subset of real space $\mathbb{R}^{2}$ and let $\partial \Omega$ be a piecewise smooth boundary of the domain $\Omega$ which is consisted of two parts; $\Gamma_{D}$ and $\Gamma_{N}$. Here, the displacement filed $\hat{u}_{i}(i=1,2)$ is prescribed on the Dirichlet boundary $\Gamma_{D}$ and surface traction $t_{i}$ is assigned on the Neumann boundary $\Gamma_{N}$. To simplify the analysis, we employ a two-dimensional rectangular domain $\Omega$ which is consisted of homogeneous and isotropic linear elastic material. Figure 1 shows a schematic illustration of the present model. As seen here, our model includes only one slip system whose Burgers vector is parallel to the $x_{1}$ direction. In order to express discontinuous displacement due to the dislocations, we introduce a set of interfaces $\bar{\Gamma}_{D}=\bigcup_{\alpha=1}^{m} \bar{\Gamma}_{D}^{\alpha}$ representing the slip plane of $\alpha$-th dislocation and jump conditions $\left[u_{i}\right]_{\bar{\Gamma}_{D}^{\alpha}}=b_{i}^{\alpha} \quad$ representing the Burgers vector. Such a discontinuous displacement due to dislocations induces elastic fields.

According to the theory of linear elasticity, we introduce the strain tensor $\epsilon_{i j}$ defined by

$$
\epsilon_{i j}=\frac{1}{2}\left(u_{i, j}+u_{j, i}\right)
$$

where $u_{i, j}$ is partial derivative of the displacement function; $u_{i, j}=\partial u_{i} / \partial x_{j}$. Here and after, we use a lower-case letter to represent a spatial direction in $\mathbb{R}^{2}$. We also employ the summation convention for repeated lower-case indices. The elastic constants tensor $C_{i j k l}$ is written in the following form

$$
C_{i j k l}=\lambda \delta_{i j} \delta_{k l}+\mu\left(\delta_{i k} \delta_{j l}+\delta_{i l} \delta_{j k}\right),
$$

where $\lambda$ and $\mu$ are Lamé constants and $\delta_{i j}$ is Kronecker delta. The strain energy density $\mathcal{W}$ of the domain $\Omega$ is defined as the inner product of $\epsilon_{i j}$ and stress tensor $\sigma_{i j}=C_{i j k l} \epsilon_{k l}$ such that

$$
\mathcal{W}=\frac{1}{2} \sigma_{i j} \epsilon_{i j}
$$

Finally, we introduce the strain energy functional $W$ as an integration of the energy density $\mathcal{W}$ over the domain $\Omega$ excluding the interfaces $\bar{\Gamma}_{D}$ such that

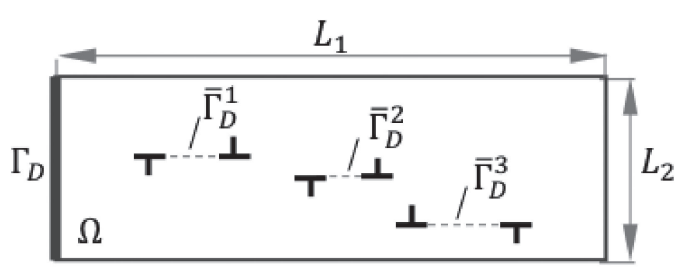

\section{$\perp$ Edge dislocation}

Fig. 1 Schematic illustration on the elastic domain $\Omega$ and edge dislocations. $\Gamma_{\mathrm{D}}$ denotes the Dirichlet boundary where displacement is fixed. $\bar{\Gamma}_{D}^{\alpha}$ represents an interface on a slip plane of $\alpha$-th dislocation. To simplify the analysis, we set $L_{1} / L_{2}=3$.

$$
W\left[u_{i}\right]=\int_{\Omega \backslash \bar{\Gamma}_{D}} \mathcal{W} d V=\int_{\Omega \backslash \bar{\Gamma}_{D}} \frac{1}{2} \sigma_{i j} \epsilon_{i j} d V .
$$

According to the variational principle for the strain energy functional $W\left[u_{i}\right]$, we obtain the stress equilibrium equation in a weak form such that

$$
\int_{\Omega \backslash \bar{\Gamma}_{D}} C_{i j k l} h_{i, j} \epsilon_{k l} d V=0,
$$

where $h_{i}$ is the test function which satisfies $h_{i}=0$ on $\Gamma_{D}$ and $\bar{\Gamma}_{D}$. This is the theoretical framework of the present study.

\subsection{Peierls-Nabarro dislocation model and XIGA}

In the present analysis, elastic fields are induced by discontinuous displacement due to the edge dislocations. Hence, to solve the stress equilibrium equation (5), we need a numerical scheme which is also suitable to represent both the discontinuous displacement and resulting elastic fields. In the present study, we use the extended isogeometric analysis (XIGA). XIGA is a generalization of IGA which solve the weak form stress equilibrium equation (5) using a linear combination of NURBS and enrichment functions. ${ }^{21-24)} \mathrm{A}$ characteristic feature of IGA is that it uses the NURBS functions both for the representation of a domain $\Omega$ and for the basis function of weak form analysis. ${ }^{25,26)}$ In other words, IGA is a Galerkin method which uses NURBS as the basis functions. On the other hand, XIGA introduces additional enrichment functions into the basis function in order to satisfy the discontinuous displacement conditions at the slip plane interfaces inside the medium.

Let us first introduce a two-dimensional parameter space defined by $\hat{\Omega}=\left\{\left(t_{1}, t_{2}\right) \mid 0<t_{1}<1,0<t_{2}<1\right\}$, where $t_{1}$ and $t_{2}$ are independent variables which run on the parameter space. The physical space $\Omega$ is obtained from the NURBS map $x_{i}: \hat{\Omega} \rightarrow \Omega$ such that

$$
x_{i}\left(t_{1}, t_{2}\right)=\sum_{I=1}^{m} N_{I}\left(t_{1}, t_{2}\right) A_{I i},
$$

where $N_{I}=N_{I}\left(t_{1}, t_{2}\right)$ is the $I$-th NURBS function defined on $\hat{\Omega}$ and $A_{I i}$ is the $i$-th component of the control point for the $I$-th NURBS function $N_{I}$. After choosing a suitable set of control points, we can express geometrically exact shape of the domain $\Omega$ using NURBS map (6).

Let $S\left(\bar{\Gamma}_{D}^{\alpha}\right)$ be the sets of indices of NURBS functions which are non-zero on the slip plane interface $\bar{\Gamma}_{D}^{\alpha}$. This set for each dislocation is obtained from the quadrilateral fourpoint mesh. ${ }^{27)}$ We approximate the displacement field $u_{i}$ and test function $h_{i}$ by linear combinations of NURBS functions such that

$$
\begin{aligned}
& u_{i}=\sum_{I=1}^{n} N_{I}\left(t_{1}, t_{2}\right) a_{I i}+\sum_{\alpha=1}^{m} \sum_{J \in S\left(\bar{\Gamma}_{D}^{\alpha}\right)} N_{J}\left(t_{1}, t_{2}\right) b_{J i}^{\alpha} \psi^{\alpha}\left(x_{1}, x_{2}\right), \\
& h_{i}=\sum_{I=1}^{n} N_{I}\left(t_{1}, t_{2}\right) c_{I i}+\sum_{\alpha=1}^{m} \sum_{J \in S\left(\bar{\Gamma}_{D}^{\alpha}\right)} N_{J}\left(t_{1}, t_{2}\right) d_{J i}^{\alpha} \psi^{\alpha}\left(x_{1}, x_{2}\right),
\end{aligned}
$$

where $a_{I i}, b_{J i}^{\alpha}, c_{I i}$ and $d_{I i}^{\alpha}$ are the real-valued coefficients of NURBS functions and $\psi^{\alpha}=\psi^{\alpha}\left(x_{1}, x_{2}\right)$ is a normalized function which represents the core shape of the $\alpha$-th 
dislocation. Roughly speaking, the first term in the right hand side of eq. (7) represents the elastic displacement and that of the second term is the discontinuous displacement due to the edge dislocations. The same holds for the test function given in eq. (8).

Some of the coefficients in eqs. (7) and (8) are determined from the Dirichlet boundary condition and interface conditions, i.e., $u_{i}=\hat{u}_{i}$ on $\Gamma_{D}$ and $h_{i}=0$ and $\left[u_{i}\right]_{\bar{\Gamma}^{\alpha}}=b_{i}^{\alpha}$ for all $\alpha$ on $\bar{\Gamma}_{D}$. Using the partition of unity of the NURBS functions, ${ }^{26)}$ we obtain

$$
\begin{aligned}
& b_{J i}^{\alpha}\left[\sum_{J \in S\left(\bar{\Gamma}_{D}^{\alpha}\right)} N_{J} \psi^{\alpha}\right]_{\bar{\Gamma}_{D}^{\alpha}}=b_{J i}^{\alpha}\left[\psi^{\alpha}\right]_{\bar{\Gamma}_{D}^{\alpha}}=b_{i}^{\alpha}, \\
& d_{J i}^{\alpha}\left[\sum_{J \in S\left(\bar{\Gamma}_{D}^{\alpha}\right)} N_{J} \psi^{\alpha}\right]_{\bar{\Gamma}_{D}^{\alpha}}=d_{J i}^{\alpha}\left[\psi^{\alpha}\right]_{\bar{\Gamma}_{D}^{\alpha}}=0,
\end{aligned}
$$

along the interface $\bar{\Gamma}_{D}^{\alpha}$. Clearly, $d_{J i}^{\alpha}=0$ satisfies eq. (10) for any function $\psi^{\alpha}$. Inserting $d_{J i}^{\alpha}=0$ in eq. (8), we obtain $h_{i}=\sum_{I=1}^{n} N_{I}\left(t_{1}, t_{2}\right) c_{I i}$. In the present study, we restrict our consideration on a single slip system for the $x_{1}$ direction and, therefore, $b_{i}^{\alpha}=\left(b_{1}^{\alpha}, 0\right)$ and $b_{J i}^{\alpha}=(b, 0)$. Then, eq. (9) can be rewritten as $\left[\psi^{\alpha}\right]_{\Gamma_{D}^{\alpha}}=b_{1}^{\alpha} / b$. In other words, $\psi^{\alpha}$ can be determined by the discontinuous displacement $b_{1}^{\alpha}$ on each $\bar{\Gamma}_{D}^{\alpha}$.

Let us express the position of the $\alpha$-th dislocation by $\left(x_{1}^{\alpha}, x_{2}^{\alpha}\right)$. In this study, we employ Peierls-Nabarro dislocation model as it excludes the stress singularity at the dislocation core. $^{28)}$ Then, $b_{1}^{\alpha} / b\left(x_{1}-x_{1}^{\alpha}\right)$ represents the normalized distribution function of Burgers vector along $\bar{\Gamma}_{D}^{\alpha}$ such that

$$
\frac{b_{1}^{\alpha}}{b}\left(x_{1}-x_{1}^{\alpha}\right)=\frac{1}{2}+\frac{1}{\pi} \tan ^{-1}\left(\frac{x_{1}-x_{1}^{\alpha}}{\zeta}\right) .
$$

Finally, the function $\psi^{\alpha}$ of Peierls-Nabarro model is given in a form of separation of variable such that

$$
\psi^{\alpha}\left(x_{1}, x_{2}\right)=\frac{b_{1}^{\alpha}}{b}\left(x_{1}-x_{1}^{\alpha}\right) H\left(x_{2}-x_{2}^{\alpha}\right),
$$

where $H\left(x_{2}-x_{2}^{\alpha}\right)$ is the Heaviside step function;

$$
H\left(x_{2}-x_{2}^{\alpha}\right)=\left\{\begin{array}{ll}
1 & x_{2}-x_{2}^{\alpha} \geq 0 \\
0 & x_{2}-x_{2}^{\alpha}<0
\end{array} .\right.
$$

The parameter $\zeta$ in eq. (11) plays an important role in the present modeling; it expresses the half width of the dislocation distribution function $b_{1}^{\alpha} / b\left(x_{1}\right)$. For the case of an edge dislocation, the parameter is expressed using the materials constants such that $\zeta=a / 2(1-v)$, where $a$ is the atomic spacing underlying the domain $\Omega$ and $v$ is the Poisson ratio. ${ }^{29)}$ Figure 2 shows the normalized dislocation distribution function $b_{1}^{\alpha} / b\left(x_{1}\right)$ for $\zeta=0.02,0.04,0.06,0.08$ and 0.10 . As seen in a following section, we set $\zeta=1 / 150 \approx$ 0.067 in the numerical analysis.

The partial derivative of eqs. (7) and (8) reads

$$
\begin{aligned}
u_{i, j}= & \sum_{I=1}^{n} \frac{\partial N_{I}}{\partial t_{k}}\left(\frac{\partial x_{j}}{\partial t_{k}}\right)^{-1} a_{I i} \\
& +\sum_{\alpha=1}^{m} \sum_{J \in S\left(\bar{\Gamma}_{D}^{\alpha}\right)} b_{J i}^{\alpha}\left(\frac{\partial N_{J}}{\partial t_{l}}\left(\frac{\partial x_{j}}{\partial t_{l}}\right)^{-1} \psi^{\alpha}+N_{J} \frac{\partial \psi^{\alpha}}{\partial x_{j}}\right),
\end{aligned}
$$

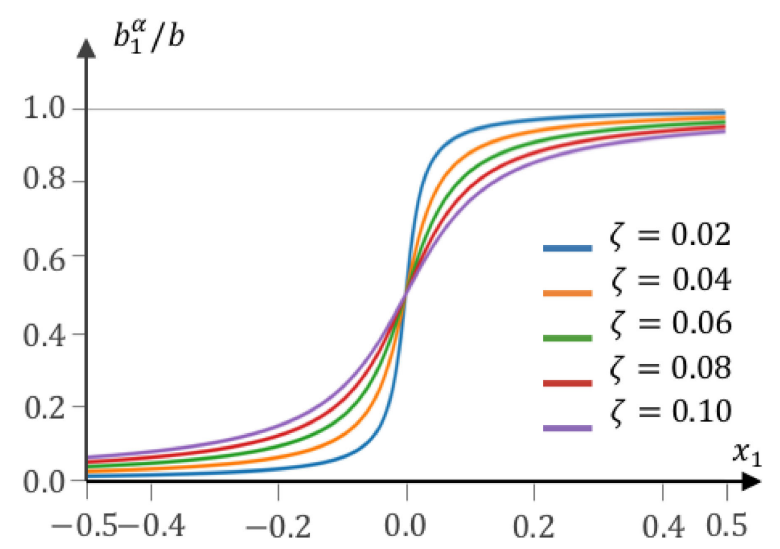

Fig. 2 Distribution of Burgers vector around the core of an edge dislocation with respect to the $x_{1}$ direction. The core width depends on the parameter $\zeta$.

$$
h_{i, j}=\sum_{I=1}^{n} \frac{\partial N_{I}}{\partial t_{k}}\left(\frac{\partial x_{j}}{\partial t_{k}}\right)^{-1} c_{I i},
$$

where $\left(\partial x_{j} / \partial t_{k}\right)^{-1}$ is obtained directly from eq. (6). Substituting eqs. (14) and (15) into (5), we obtain

$$
\sum_{I=1}^{n} \sum_{J=1}^{n} c_{I j}\left(K_{I j J k} a_{J k}+F_{I j}\right)=0,
$$

where $K_{I j J k}$ and $F_{I j}$ are defined as follows;

$$
\begin{gathered}
K_{I i J k}=\int_{\hat{\Omega}} C_{i j k l} \frac{\partial N_{I}}{\partial t_{m}}\left(\frac{\partial x_{j}}{\partial t_{m}}\right)^{-1} \frac{\partial N_{J}}{\partial t_{n}}\left(\frac{\partial x_{l}}{\partial t_{n}}\right)^{-1} d V, \\
F_{I j}=\int_{\hat{\Omega}} C_{i j k l} \frac{\partial N_{I}}{\partial t_{p}}\left(\frac{\partial x_{j}}{\partial t_{p}}\right)^{-1} \\
\times \sum_{\alpha=1}^{m} \sum_{J \in S\left(\bar{\Gamma}_{D}^{\alpha}\right)}\left(\frac{\partial N_{J}}{\partial t_{q}}\left(\frac{\partial x_{l}}{\partial t_{q}}\right)^{-1} \psi^{\alpha}+N_{J} \frac{\partial \psi^{\alpha}}{\partial x_{l}}\right) d V .
\end{gathered}
$$

Equation (16) must be satisfied for any $c_{I j}$. This condition reads a system of linear algebraic equations for unknown coefficients $a_{J k}$ such that

$$
K_{I j J k} a_{J k}=-F_{I j} .
$$

The total degrees of freedom of eq. (19) depends on the number of NURBS basis functions; $\chi=2 n$.

\section{Results and Discussion}

\subsection{Simulation conditions}

As shown in Fig. 1, we consider the two-dimensional rectangular domain $\Omega$ defined by $\Omega=\left\{\left(x_{1}, x_{2}\right) \mid 0<x_{1}<\right.$ $\left.L_{1}, 0<x_{2}<L_{2}\right\}$. Peierls-Nabarro model includes atomic spacing $a$ in the core width parameter $\zeta$. Using the absolute length scale, the size of the medium is normalized such that $L_{1}=300 a$ and $L_{2}=100 a$ with $a=0.01$. Similarly, the coefficient $b$ which defines the strength of the Burgers vector is defined using the parameter $a$ by $b=a$. Lamé constants are also normalized to $\lambda=\mu=1$ so as to satisfy the Cauchy solid condition with the Poisson ratio $v=\lambda / 2(\lambda+\mu)=$ 0.25 . Consequently, the core width parameter $\zeta$ becomes $\zeta=1 / 150 \approx 0.067$. The Dirichlet boundary condition is set on the left surface of the rectangular domain $\Omega$ such that $\hat{u}_{1}=\hat{u}_{2}=0$ on $x_{1}=0$. The remaining boundary $\partial \Omega \backslash \Gamma_{\mathrm{D}}$ is 
classified as Neumann boundary, which is free from any surface traction; $t_{i}=\sigma_{i j} n_{j}=0$.

The parameter domain $\hat{\Omega}=(0,1) \times(0,1)$ is divided into subdomains by the open and uniform knot vectors; the numbers of knots are 1,497 in the $x_{1}$ and 497 in the $x_{2}$ directions, respectively. The polynomial orders of B-spline functions are $p_{1}=p_{2}=3$. It indicates that the NURBS basis function is $C^{2}$ class in the entire domain $\Omega$. The weights of control points are taken to be unity. Consequently, the numbers of B-spline basis functions in each direction are $n_{1}=1500$ and $n_{2}=500$, leading to the total number of NURBS basis function to be $n=n_{1} n_{2}=750,000$. Hence, the degree of freedom is $\chi=1,500,000$.

\subsection{Microstructure of ortho kink deformation}

As mentioned in a previous section, Hess and Barrett explained the kink structure on the basis of the distribution of edge dislocations. $\left.{ }^{6}\right)$ According to the modeling, there are two different kinds of kink structures called ortho kink and ridge kink both of which are found in experiments on $\mathrm{Zn}^{6,8}$ ) and LPSO-Mg alloys. ${ }^{30)}$

Let us first investigate the ortho kink structure. Figure 3(a) shows the configuration of dislocations for an ortho kink structure. We assume that five pairs of edge dislocations are emitted from five dislocation sources which are aligned at the center of domain $\Omega$ with respect to the $x_{1}$ direction. Essentially the similar dislocation configuration is considered in Fig. 3(b). In this case, however, dislocations have the opposite signs against those in Fig. 3(a). Macroscopic deformations due to the configurations in (a) and (b) are respectively shown in Figs. 3(c) and (d). Note that the deformations are magnified by a factor of 10 . As expected from the Hess-Barrett model, the macroscopic shapes show the sharp inflections at the lines where dislocations align. This is a characteristic morphology of kink deformation. The color in the figures show the magnitude of elastic spin $\theta$ which is defined by the following form

$$
\theta=\epsilon_{3 i j} \omega_{j i}=\frac{1}{2}\left(u_{2,1}-u_{1,2}\right)
$$

Here $\epsilon_{i j k}$ is the alternating tensor. Roughly speaking, $\theta$ is the measure of the lattice rotation due to the deformation. It is
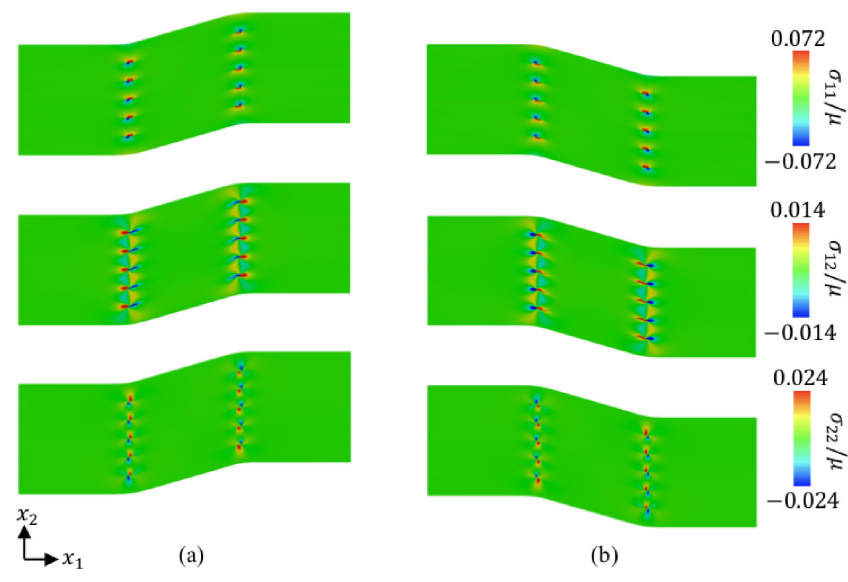

(b)

Fig. 4 Stress fields obtained from the dislocation configurations given in Figs. 3. In order to avoid the large stress concentration at the dislocation cores, we restricted the range of color distributions to be $20 \%$ of the actual numerical result.

evident from the numerical analysis that the sign of dislocations determines the direction of elastic spin $\theta$. We can also see from the figures that the spin is highly localized within the dislocation arrays, namely within the kink boundaries. It also shows that distribution of rotation is uniform. These features agree well with the experimental results. $^{6,8)}$

Figures 4(a) and (b) show internal stress fields of $\sigma_{11}, \sigma_{22}$ and $\sigma_{12}$ obtained from the dislocation configuration given in Figs. 3(a) and (b). Note that the stress components are normalized by shear modulus $\mu$. Unlike the elastic spin $\theta$ shown in Figs. 3(c) and (d), the stress fields are not distributed within the kink boundaries but localized only around the cores of dislocations. In addition, this feature is independent of the stress components. This result implies that stress field around the ortho kink boundary is similar to low angle boundary made by polygonization of edge dislocations. In other words, strengthening due to the ortho kink structure would be comparable to conventional grain boundary refinement.

\subsection{Microstructure of ridge kink deformation}

Another kink structure we are interested in is the ridge (a)

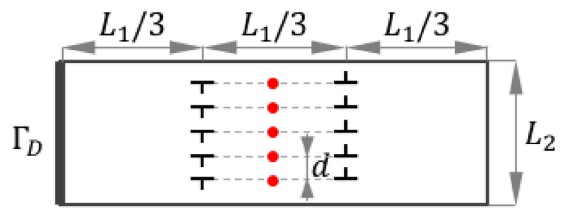

(c)

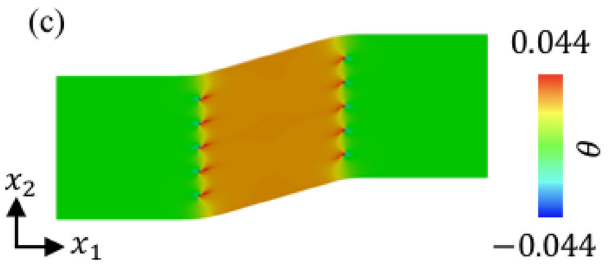

(b)

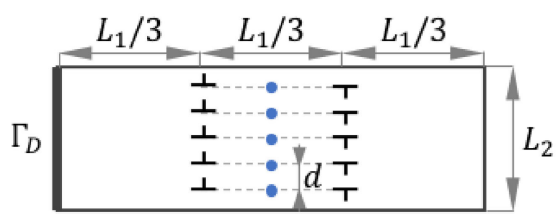

(d)

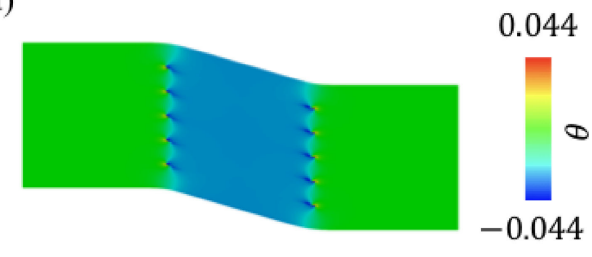

Fig. 3 (a) and (b) show dislocation configurations for an ortho kink structure. The red and blue circles in the figures show the dislocation sources assigned respectively to positive and negative. Resulting deformation fields are shown in (c) and (d), where the color distribution shows elastic $\operatorname{spin} \theta$. In order to avoid the large spin concentration at the dislocation cores, we restricted the range of color distribution to be $60 \%$ of actual numerical result. 
(a)

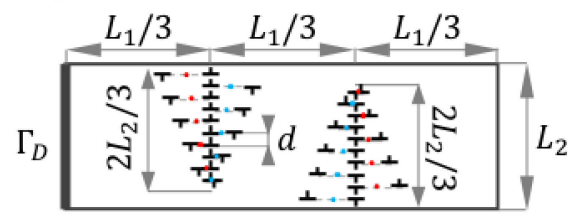

(c)

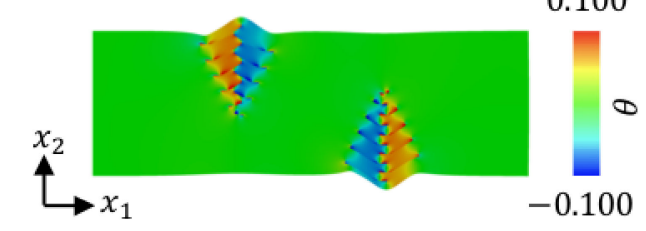

(b)

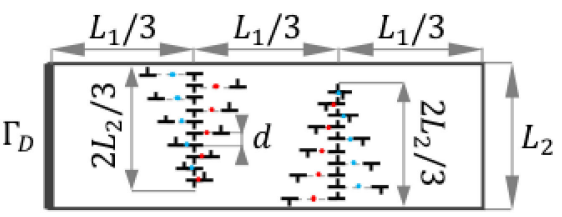

(d)

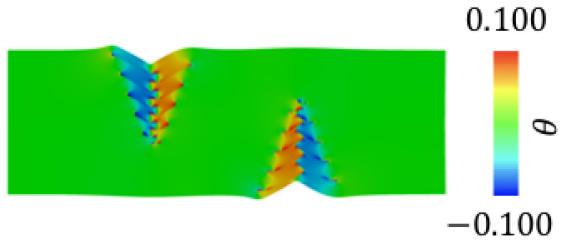

Fig. 5 (a) $C_{2}^{+}$and (b) $C_{2}^{-}$show dislocation configurations for ridge kink structures. The red and blue circles in the figures show the dislocation sources assigned respectively to positive and negative. Resulting deformation fields are shown in (c) and (d), where the color distribution shows the distribution of elastic spin $\theta$. Although maximum values of spin reach $\theta= \pm 0.190$ around the cores of the dislocations, the color distribution is restricted to $\theta= \pm 0.100$.

kink. Following the Hess-Barrett model, ${ }^{6)}$ we consider the configurations of dislocations as illustrated in Fig. 5(a). In this case, ten pairs of dislocations are considered; five pairs are emitted from the positive sources (red circles) while the remaining five pairs come from the negative sources (blue circles). Essentially the same dislocation configurations are shown in Fig. 5(b). Only the difference here is the sign of Burgers vector. For the sake of convenience, we denote the two configurations by $C_{2}^{+}$and $C_{2}^{-}$. Figures 5(c) and (d) show the macroscopic deformation obtained from the dislocation configurations $C_{2}^{+}$and $C_{2}^{-}$. Note that the deformation is magnified by a factor of 10 and the color contour represents the distribution of the elastic spin $\theta$. As seen in (c), the macroscopic shape shows sharp extrusions on the top and bottom surfaces of the domain. This is a typical feature of ridge kink structure. ${ }^{6,8,30}$ We also see that each extrusion is consisted of two sub-regions which have the opposite sign of the elastic $\operatorname{spin} \theta$. In addition, the spins are highly localized within the ridge kink boundaries. Comparing the result to Figs. 3(a) and (b), this structure is explained by combining the two ortho kinks which have the opposite sign of spin. In a previous study, Lei and Nakatani conducted numerical analysis for two-dimensional atomistic system and found the formation of extrusion-type ridge kink structure under compressive loading. ${ }^{19)}$ They also revealed that the ridge kink is consisted of two sub-regions that have the opposite rotational displacement localized within the kink boundaries. These features agree qualitatively well with the present numerical analysis. We therefore conclude that the extrusion type ridge kink structure is explained by a special configuration of edge dislocations, i.e., $C_{2}^{+}$type configuration.

The dislocation configuration of $C_{2}^{-}$is obtained by simply reversing the signs of edge dislocations in $C_{2}^{+}$. Hence, the resulting macroscopic shape would be the opposite one; it becomes intrusion-type both on the top and bottom surfaces of the domain. In fact, we can confirm the intrusion-type ridge kink structure in Fig. 5(d). Although the two kink structures $C_{2}^{ \pm}$have essentially the same configuration of dislocations, there is a remarkable difference between the structures. The extrusion-type ridge kink is energetically favorable under a compression while the intrusion-type kink is favorable under an extension since they compensate the volumetric change due to the external loads. In the actual experiments, extrusion-type ridge kink appears frequently but the observation of intrusion-type is limited. This experimental evidence implies that the formation of ridge kink structure is triggered by mechanical instability, such as elastic buckling, as it appears only in the compressive loading.

Figure 6(a) shows another configuration of dislocations denoted by $D_{1}^{+}$. In this case, the ten pairs of edge dislocations are emitted from ten dislocation sources that have the same sign, say red circles. Consequently, at the middle part of a ridge kink, edge dislocations of different signs are aligned and they counteract the displacement as well as the stress fields with each other. Hence, unlike the previous two cases of $C_{2}^{ \pm}$, the resulting macroscopic deformation has uniform spin within the kink boundaries (see Fig. 6(c)). Figures 6(b) and (d) are similar configurations of dislocations except for the signs of Burgers vectors. According to the macroscopic morphology and elastic spin within the kink boundaries, the two configurations $D_{1}^{ \pm}$are similar to ortho kink structures shown in Figs. 3. In some way, the kink structures $D_{1}^{ \pm}$are incomplete ortho kink which are terminated within the domain. As shown in (b) and (d), such an incomplete deformation requires the complementary rotation outside the kink regions due to the requirement from the strain compatibility condition.

\subsection{Strengthening by ridge kink}

Numerical analysis given in previous sections revealed that the morphologies of ridge kinks are explained from the arrays of edge dislocations as suggested by Hess-Barrett. ${ }^{6}$ ) The remaining issue that we want to address here is the understanding of strengthening mechanism by the ridge kinks. Obviously, the most important information is included in the stress field around the kink boundary since dislocations interact through stress fields in the elastic medium. ${ }^{17)}$ Figure 7 shows the internal stress fields obtained from the dislocation configurations $C_{2}^{+}$and $C_{2}^{-}$. Similar to the cases of ortho kinks, most of them are localized near the cores of dislocations. However, the stress components $\sigma_{22}$ shows 
(a)

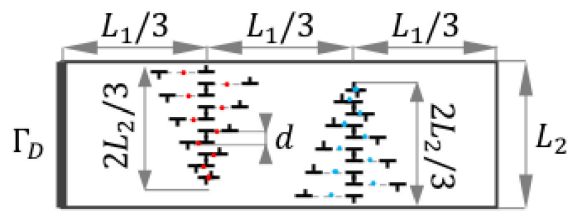

(c)

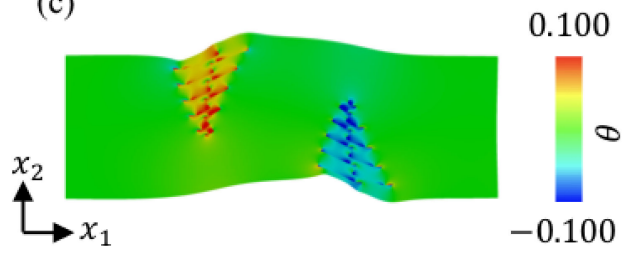

(b)

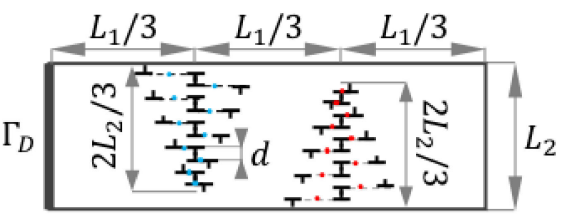

(d)

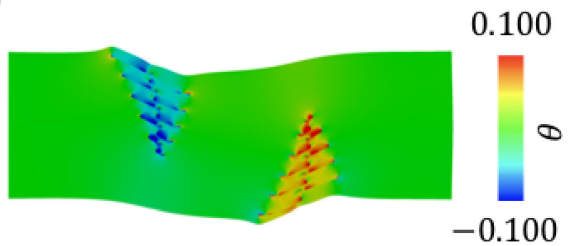

Fig. 6 (a) $D_{1}^{+}$and (b) $D_{1}^{-}$show dislocation configurations for ridge kink structures. The red and blue circles in the figures show the dislocation sources assigned respectively to positive and negative. Resulting deformation fields are shown in (c) and (d), where the color distribution shows the distribution of elastic spin $\theta$. Although maximum values of spin reach $\theta= \pm 0.228$ around the cores of the dislocations, the color distribution is restricted to $\theta= \pm 0.100$.
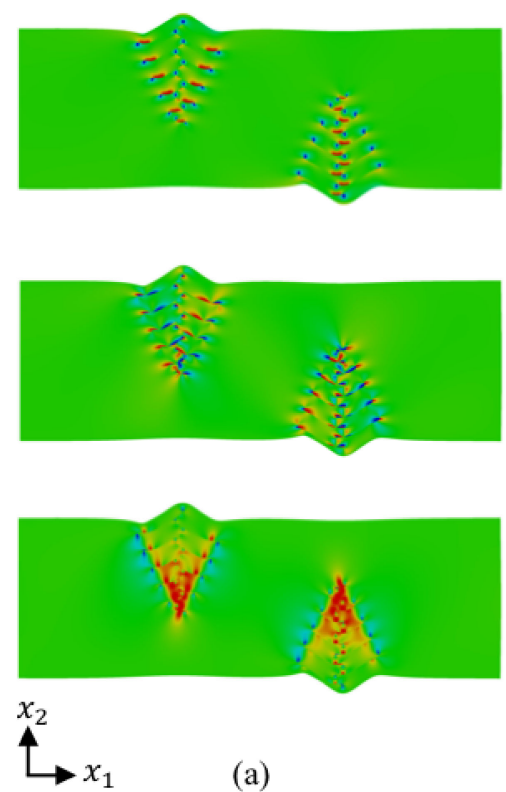
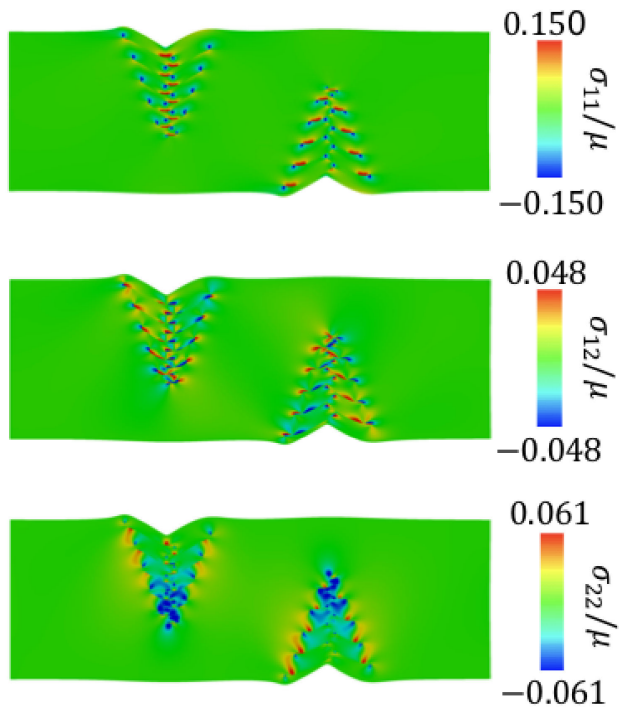

(b)

Fig. 7 Stress fields obtained from (a) $C_{2}^{+}$configuration and (b) $C_{2}^{-}$configuration. Although, most of the stress components are localized near the cores of dislocations, only the stress component $\sigma_{22}$ shows broad distribution within the kink boundaries. In order to avoid the large stress concentration at the dislocation cores, we restricted the range of color distributions to be $20 \%$ of the actual numerical result.

broad distribution through the inner region of kink boundaries. Especially, the stress concentration is notable around the tip of ridge kink structures. Recently, Inamura investigated kink deformation in two-dimensional continuum system on the basis of rank-one connection. ${ }^{31)}$ From the kinematical analysis, he concluded that there exists disclination at the tip of ridge kink structures. Comparing the result to present numerical analysis, the $\sigma_{22}$ stress concentration found in $C_{2}^{ \pm}$might be related to the formation of disclination. Such a long-range stress field would affect the motion of subsequent dislocations during the plastic deformation. It explains a part of kink strengthening mechanism.

Internal stress fields for dislocation configurations $D_{1}^{+}$and $D_{1}^{-}$are summarized in Figs. 8. Unlike the previous case, the stress concentration is insignificant and no additional strengthening is expected compared with the conventional low-angle grain boundary refinement. On the other hand, these configurations show notable distortion around the kink boundaries. As mentioned in the previous section, the ridge kinks in $D_{1}^{ \pm}$are terminated within the domain and therefore induce the complementary rotation outside the kink boundaries. Obviously, such a rotation influences on the apparent yield stress due to the change in Schmid factor and might be effective for strengthening of kink-forming highly anisotropic materials.

The last thing that we need to address here is the constitutive equation. Within the framework of classical linear elasticity, only the symmetric part of displacement gradient, namely the strain tensor, is considered in the constitutive equation (3). In other words, rotational displacement produces no stress fields. This situation might be different if we employ a non-classical constitutive equation such as strain gradient elasticity or micropolar elasticity. ${ }^{32,33)}$ In other words, the stress field analysis within the classical elasticity might underestimate the strengthening mechanism due to kink structure. 

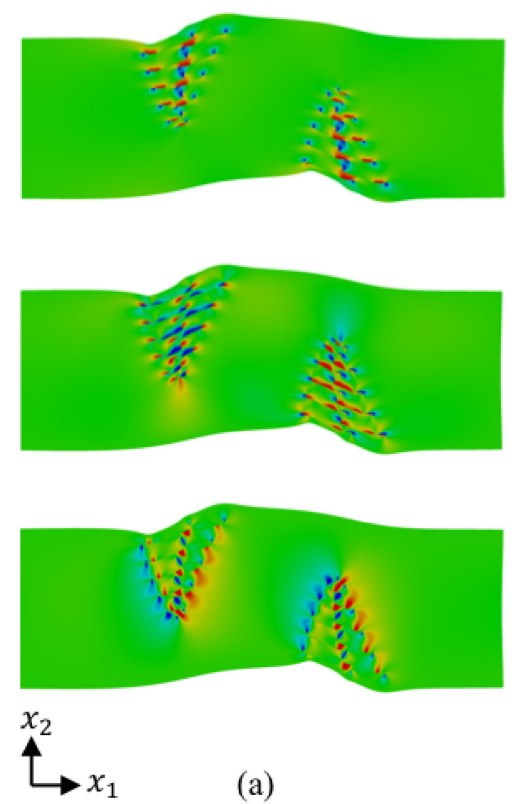

(a)
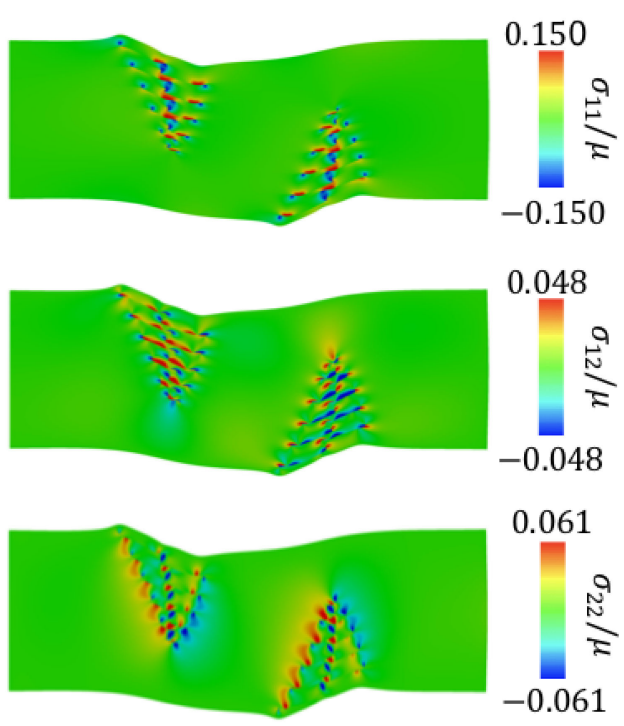

(b)

Fig. 8 Stress fields obtained from (a) $D_{1}^{+}$configuration and (b) $D_{1}^{-}$configuration. All of the stress components are localized near the cores of dislocations. In order to avoid the large stress concentration at the dislocation cores, we restricted the range of color distributions to be $20 \%$ of the actual numerical result.

\section{Conclusion}

In the present study, we investigated the macroscopic deformation as well as internal stress fields due to ortho and kink structures on the basis of Hess-Barrett edge dislocation model. Our formulation is based on the linear elastic continuum mechanics solved numerically by XIGA. Conclusion of the present study is summarized as follows:

(1) We consider two-dimensional continuum domain $\Omega$ which includes edge dislocations expressed by PeierlsNabarro model. Weak form stress equilibrium equation is derived from the variational principle and solved numerically using XIGA, i.e., Galerkin method which uses NURBS as the basis functions.

(2) Numerical analysis for ortho kink structure revealed that elastic spin is uniformly distributed within the kink boundaries. On the other hand, stress fields are highly localized around the cores of the dislocations. Such a dislocation microstructure is similar to low angle grain boundary and which implies that the resulting strengthening is explained by conventional Hall-Petch effect.

(3) Numerical analysis for $C_{2}^{+}$type configurations of dislocations revealed that the macroscopic deformation becomes extrusion type ridge kink structure. On the other hand, $C_{2}^{-}$type configurations showed the opposite manner, i.e., intrusion-type ridge kink. Although the two ridge kinks have dual configurations, only extrusion-type kink appears in the actual experiments. This result implies that the formation of ridge kink is triggered by elastic instability such as buckling.

(4) Numerical analysis for $D_{1}^{ \pm}$configurations revealed that their spin distributions are similar to ortho kink structures. Only the difference is the termination of kink structure and which induces the complementary rotation outside the kink boundaries.
(5) Stress field analysis revealed that there exists notable stress concentration on $\sigma_{22}$ components in $C_{2}^{+}$ configurations. Comparing the previous results on kinematical analysis, it might be related to the formation of disclination at the tip of ridge structures. Crystallographic rotation due to the complementary rotation in $D_{1}^{ \pm}$is another effective strengthening mechanism as it influences on Schmid factor in a local region.

(6) Stress field analysis on the basis of classical elasticity might underestimate of the actual stress fields as it excludes the rotational contributions. It would be worthwhile to introduce a generalized constitutive equation such as strain gradient elasticity or micropolar elasticity.

\section{Acknowledgments}

This study was supported by JSPS KAKENHI for Scientific Research on Innovative Areas "MFS Materials Science (Grant Numbers JP18H05481)". The authors acknowledge Prof. T. Fujii (Tokyo Institute of Technology) for his valuable advice and comments.

\section{REFERENCES}

1) E. Abe, Y. Kawamura, K. Hayashi and A. Inoue: Acta Mater. 50 (2002) 3845-3857.

2) A. Inoue, Y. Kawamura, M. Matsushita, K. Hayashi and J. Koike: J. Mater. Res. 16 (2001) 1894-1900.

3) Y. Kawamura, K. Hayashi, A. Inoue and T. Masumoto: Mater. Trans. 42 (2001) 1172-1176.

4) K. Hagihara, Z. Li, M. Yamasaki, Y. Kawamura and T. Nakano: Acta Mater. 163 (2019) 226-239.

5) E. Orowan: Nature 149 (1942) 643-644.

6) J.B. Hess and C.S. Barrett: Trans. Metall. AIME 185 (1949) 599-606.

7) D.C. Jillson: Trans. Metall. AIME 188 (1950) 1009-1018.

8) J.J. Gilman: J.O.M. 6 (1954) 621-629. 
9) S. Yoshimoto, M. Yamasaki and Y. Kawamura: Mater. Trans. 47 (2006) 959-965.

10) M. Noda, T. Mayama and Y. Kawamura: Mater. Trans. 50 (2009) 2526 2531.

11) D.A. Zaukelies: J. Appl. Phys. 33 (1962) 2797-2803.

12) B. Marshall: Nature 210 (1966) 1249-1251.

13) T.B. Anderson: Nature 213 (1967) 54-55.

14) F. Hörz: J. Geophys. Res. 75 (1970) 965-977.

15) T.J. Nizolek, M.R. Begley, R.J. McCabe, J.T. Avallone, N.A. Mara, I.J. Beyerlein and T.M. Pollock: Acta Mater. 133 (2017) 303-315.

16) G.W. Hunt, M.A. Peletier and M.A. Wadee: J. Struct. Geol. 22 (2000) 669-681.

17) L. Shaofan and W. Gang: Introduction to Micromechanics and Nanomechanics, (World Scientific Publishing, Toh Tuck Link, 2008).

18) R. Matsumoto, M. Uranagase and N. Miyazaki: Mater. Trans. 54 (2013) 686-692.

19) X.-W. Lei and A. Nakatani: J. Appl. Mech. 82 (2015) 071016.

20) R. Peierls: Proc. Phys. Soc. 52 (1940) 34-37.

21) S.S. Ghorashi, N. Valizadeh and S. Mohammadi: Int. J. Numer Methods Eng. 89 (2012) 1069-1101.

22) R. Gracie, G. Ventura and T. Belytschko: Int. J. Numer. Methods Eng.
69 (2007) 423-441.

23) J. Oswald, R. Gracie, R. Khare and T. Belytschko: Comput. Methods Appl. Mech. Eng. 198 (2009) 1872-1886.

24) J. Oswald, E. Wintersberger, G. Bauer and T. Belytschko: Int. J. Numer. Methods Eng. 85 (2011) 920-938.

25) T.J.R. Hughes, J.A. Cottrell and Y. Bazilevs: Comput. Methods Appl. Mech. Eng. 194 (2005) 4135-4195.

26) J.A. Cottrell, T.J.R. Hughes and Y. Bazilevs: Isogeometric Analysis: Toward Integration of CAD and FEA, (Wiley Publishing, West Sussex, 2009).

27) B. Gernot and B. Stéphane, eds.: Isogeometric Methods for Numerical Simulation, (Springer, Vienna, 2015) pp. 21-120.

28) M. Lazar: Philos. Mag. 97 (2017) 3246-3275.

29) V.A. Lubarda and X. Markenscoff: Arch. Appl. Mech. 77 (2007) 147154.

30) K. Hagihara, N. Yokotani and Y. Umakoshi: Intermetallics 18 (2010) 267-276.

31) T. Inamura: Acta Mater. 173 (2019) 270-280.

32) R.D. Mindlin: Arch. Ration. Mech. Anal. 16 (1964) 51-78.

33) A.C. Eringen: Microcontinuum Field Theories, (Springer, New York, 1999) pp. 101-248. 